\title{
Corneal sensitivity and morphology of the corneal subbasal nerve plexus in primary congenital glaucoma
}

${ }^{1}$ Department of Ophthalmology, University Medical Center of Saarland UKS, Homburg/Saar, Germany

${ }^{2}$ Department of Ophthalmology, University Hospitals of Geneva HUG, Geneva, Switzerland

Correspondence:

Z Gatzioufas, Department of Ophthalmology, University Hospitals of Geneva HUG, Rue AlcideJentzer 22, 1211 Geneva, Switzerland

Tel: +41 795533878;

Fax: +41 (0)22 3828433

E-mail: zisisg@hotmail.com

Received: 20 December 2012 Accepted in revised form: 10 December 2013 Published online: 31 January 2014

\begin{abstract}
Background To quantify the corneal subbasal nerve density and the total number of nerve fibers in primary congenital glaucoma (PCG) and to evaluate their impact on corneal sensitivity. Methods Forty eyes of 26 PCG patients were compared with 40 eyes randomly selected from 40 non-glaucoma patients who populated the control group. Central corneal sensitivity (CCS) was assessed by means of Cochet-Bonnet esthesiometry. The mean subbasal nerve density and the total number of nerve fibers were quantified by laserscanning confocal microscopy. Normality of data was assessed by Kolmogorov-Smirnov testing. Differences in parameters were assessed with Student's $t$-test, while correlations with CSS were assessed with Pearson's correlation.

Results Significant differences were identified in the mean subbasal nerve density $(2108 \pm 692 \mu \mathrm{m}$ in PCG, $2642 \pm 484 \mu \mathrm{m}$ in controls, $P=0.003$ ) and in the total number of nerve fibers (12.3 \pm 4.2 in PCG, $15.4 \pm 3.1$ in controls, $P=0.02$ ). Both groups presented comparable mean CCS and tortuosity. Both groups presented strong correlations between CCS and mean nerve density ( $r=0.57$ in PCG, $r=0.67$ in controls, all $P<0.05$ ), and between CCS and total number of nerve fibers $(r=0.55$ in PCG, $r=0.56$ in controls, all $P<0.05$ ).

Conclusion PCG exerts significant changes in both the mean subbasal nerve density and the total number of nerve fibers. However, these changes do not appear to affect central corneal sensitivity.

Eye (2014) 28, 466-471; doi:10.1038/eye.2014.4; published online 31 January 2014
\end{abstract}

Z Gatzioufas ${ }^{1,2}$, G Labiris ${ }^{1}$, F Hafezi ${ }^{2}$, A Schnaidt ${ }^{1}$, B Pajic ${ }^{2}$, A Langenbucher ${ }^{1}$ and B Seitz ${ }^{1}$
Keywords: congenital glaucoma; corneal sensitivity; subbasal nerve plexus; in vivo laser-scanning confocal microscopy

Introduction

Primary congenital glaucoma (PCG) is characterized by increased intraocular pressure (IOP), enlarged corneal diameter, and marked optic-disc cupping in the first year of life. ${ }^{1}$ Elevated IOP causes enlargement of the globe (buphthalmos), resulting in breaks in the Descemet's membrane, the so-called Haab's striae. $^{2}$ Another common finding of PCG is corneal opacification or haze, while the corneal biomechanical profile is also significantly modified in PCG. ${ }^{3,4}$

PCG has been associated with mutations in the gene CYP1B1 and LTBP2m. ${ }^{5}$ Isolated dysgenesis of the trabecular meshwork has been identified as the anatomical cause of PCG, but the underlying pathophysiology is still obscure. ${ }^{6}$ Mastropasqua et $a l^{7}$ examined two adult patients with congenital glaucoma and megalocornea with the aid of a scanning slit corneal confocal microscopy and reported an abnormal, 'coil-shaped' configuration of the stromal nerve fiber network. However, alterations in the corneal subbasal nerve plexus in PCG and their potential impact on corneal sensitivity have not been investigated in a validated and quantitative manner, yet.

Laser-scanning confocal microscopy (LSCM) is a modern, noninvasive modality that allows high-resolution imaging of the cornea at the cellular level in vivo, increasing our understanding of corneal nerve anatomy in healthy and diseased human corneas. 8,9 
Recent studies analyzed the morphology of the subbasal nerve plexus in normal corneas by confocal microscopy. ${ }^{10-12}$ Moreover, the architecture of the corneal subbasal nerve plexus has been investigated, with the aid of confocal microscopy, in patients with keratoconus, Sjogren syndrome, or dry eye, as well as after penetrating keratoplasty, laser in situ keratomileusis, or photorefractive keratectomy. ${ }^{13-21}$

Within this context, the purpose of this study was to investigate the corneal sensitivity in patients with PCG and correlate the clinical findings with the corneal subbasal nerve density by use of LSCM.

\section{Patients and methods}

\section{Setting}

This prospective, observational study was conducted at the Department of Ophthalmology, University Medical Center of Saarland UKS (Homburg/Saar, Germany) during the period between May and September 2011. The Institutional Review Board of the University approved the study protocol, which adhered to the tenets of the Declaration of Helsinki and written informed consent was obtained by all participants before inclusion in the study.

\section{Participants}

Patients with PCG were recruited in a consecutiveif-eligible basis from the Glaucoma Service of the aforementioned hospital. The diagnosis of PCG was established by the documented elevated IOP ( $>21 \mathrm{~mm} \mathrm{Hg}$ ), enlarged corneal diameter $(>12.5 \mathrm{~mm})$, and abnormal cup-to-disc ratio $(\mathrm{CDR}>0.4)$ in the first year of life.

Exclusion criteria included: diabetes, AxenfeldRieger's anomaly, Peters' anomaly, previous uveitis, previous trauma, history of retinopathy of prematurity, previous corneal surgery, corneal opacification or visible corneal edema, strabismus, nystagmus, poor compliance, or fixation difficulties.

Patients with PCG were compared with a random sample of non-glaucoma subjects who populated the control group. Eligibility for participation in the control group included IOP values below $21 \mathrm{mmHg}$ in repeated measurements, $\mathrm{CDR}<0.4$, and normal visual fields as examined by Octopus 30II-perimetry (Haag-Streit AG, Koeniz, Switzerland). Exclusion criteria for the control group included significant systemic illnesses and ocular conditions, such as corneal opacity, uveitis, or history of intraocular surgery, refractive surgery, glaucoma, or retinal disease.

\section{Corneal sensitivity measurement}

Central corneal sensitivity was measured bilaterally with a Cochet-Bonnet esthesiometer (Luneau Ophthalmlogie, Chartres, France). This test mechanically stimulates corneal nerves, by pressing a retractable $6 \mathrm{~cm}$ length monofilament nylon thread of $0.12 \mathrm{~mm}$ diameter against the anterior corneal surface, shortening in steps of $1.0 \mathrm{~cm}$ if a positive response is not obtained. If a positive response was obtained, the thread was advanced by $0.5 \mathrm{~cm}$. The longest filament length resulting in a positive response was considered the corneal sensitivity threshold, which was verified two times.

\section{In vivo confocal laser-scanning microscopy}

In vivo LSCM was performed in all patients using the Heidelberg Retina Tomograph (HRT II; Heidelberg Engineering $\mathrm{GmbH}$, Heidelberg, Germany) in combination with the Rostock Cornea Module (RCM). ${ }^{22}$ This microscope uses a 670-nm laser source, a class 1 laser system, and thus, by definition, does not pose any ocular safety hazard. The eye to be examined was first anesthetized by instilling proparacain $0.5 \%$ eye drops (Ursapharm, Saarbrücken, Germany). Coupling between the patient's cornea and the contact cap was facilitated with a thin lubricant layer of Vidisic gel (Bausch \& Lomb/Dr Mann Pharma, Berlin, Germany). Digital image storage was performed using a water immersion objective ( $\times 63 / 0.95 \mathrm{~W}, 670 \mathrm{~nm}, \infty / 0$; Zeiss, Jena, Germany) with an adjustable focus plane. The HRT II/RCM provides image segments measuring $400 \times 400 \mu \mathrm{m}^{2}$ and the manufacturer quotes the transverse resolution and optical section thickness as 2 and $4 \mu \mathrm{m}$, respectively. The images of all corneal layers were captured in the central cornea. The device's z-scan depth function permitted automatic image acquisition (up to 30 images/s) while traversing a corneal volume for later three-dimensional reconstruction.

During the examination, all subjects were asked to fixate on a distance target aligned to enable examination of the central cornea. The full thickness of the central cornea was scanned using the device's section mode. This mode enables instantaneous imaging of a single area of the cornea at a desired depth. The total duration of in vivo confocal examination was approximately $10 \mathrm{~min}$ per eye, and none of the subjects experienced any visual symptoms or corneal complications as a result of the examination.

\section{Image analysis}

Only images of the central cornea were collected.

Assessment included at least eight images from each 
subject. The best-focused subbasal plexus images were considered for the analysis. An experienced observer (AS) reviewed all scans. All frames were randomized subsequently and were encoded by an independent observer (GL). For all images, a standard frame size of $300 \times 300 \mu \mathrm{m}^{2}$ was selected. An experienced examiner (ZG) analyzed the subbasal nerve plexus as described before. ${ }^{19}$ Briefly, nerve density was assessed by measuring the total length of the nerve fibers in micrometers per frame $\left(0.06 \mathrm{~mm}^{2}\right)$. The number of total nerves was also measured and it was defined as the number of all nerves, including main nerve trunks and branches in one image. Tortuosity of the subbasal nerve fibers was also evaluated, according to the method proposed by Oliveira-Soto and Efron, ${ }^{10}$ and graded from 0 to 4 as in previously published studies. ${ }^{23,24}$

\section{Statistical analysis}

Data were analyzed with the Statistical Package for Social Sciences (SPSS Inc., Chicago, IL, USA; version 17.0). All data are expressed as the mean \pm SD. Normality of data was assessed by Kolmogorov-Smirnov testing. The differences of cell densities between PCG eyes and normal controls were evaluated with Student's $t$-test. Parametric data were tested for relationships using Pearson's correlation coefficients. $P$-values $<0.05$ were considered statistically significant.

\section{Results}

Forty eyes of 26 PCG patients (12 men/14 women, $12.6 \pm 4.8$ years) and 40 eyes of 40 non-glaucoma patients (20 men/20 women, $14.2 \pm 2.6$ years) populated the PCG group and the control group, respectively. Seventy percent of the PCG eyes $(28 / 40)$ had been subjected to goniotomy and $85 \%$ of them $(34 / 40)$ were under topical antiglaucomatous therapy (carboanhydrase inhibitors and/or brimonidine eye drops). Groups presented significant differences in the best spectacle-corrected visual acuity $(P<0.001)$ and the corneal diameter $(P<0.001)$ (Table 1$)$. Central corneal thickness was significantly reduced in patients with PCG $(519 \pm 34 \mu \mathrm{m})$ compared with the control group $(566 \pm 22 \mu \mathrm{m})$ $(P<0.001)$.

Mean corneal sensitivity in patients with PCG $(5.8 \pm 0.2)$ was comparable to that in controls $(5.8 \pm 0.2)$ (Student's $t$-test, $P=0.2$ ). On the other hand, significant differences between the two groups were identified in the mean subbasal nerve density $(2108 \pm 692 \mu \mathrm{m}$ in PCG, $2642 \pm 484 \mu \mathrm{m}$ in controls, $P=0.003)$ and in the total number of nerve fibers (12.3 \pm 4.2 in PCG, $15.4 \pm 3.1$ in controls, $P=0.02$ ) (Figure 1). However, the grade of tortuosity was comparable in both groups (Table 2 ).
Table 1 Demographic and clinical data

\begin{tabular}{|c|c|c|c|}
\hline \multirow[t]{2}{*}{ Parameters } & \multicolumn{2}{|c|}{ Study groups } & \multirow[t]{2}{*}{ P-values } \\
\hline & $P C G(\mathrm{n}=40)$ & $\begin{array}{l}\text { Controls } \\
(\mathrm{n}=40)\end{array}$ & \\
\hline Age (years) & $12.6 \pm 4.8$ & $14.2 \pm 2.6$ & 0.04 \\
\hline BCVA & $0.4 \pm 0.1$ & $0.8 \pm 0.1$ & $<0.001$ \\
\hline Corneal diameter (mm) & $14.1 \pm 1.2$ & $11.4 \pm 0.6$ & $<0.001$ \\
\hline Corneal sensitivity (cm) & $5.8 \pm 0.2$ & $6.0 \pm 0$ & 0.2 \\
\hline
\end{tabular}

Abbreviations: BCVA, best-corrected visual acuity; PCG, primary congenital glaucoma.

Student's $t$-test was applied. $P$-values $<0.05$ were considered statistically significant.

Furthermore, both groups presented strong correlations between corneal sensitivity and mean nerve density ( $r=0.57$ in PCG, $r=0.67$ in controls, all $P<0.05$ ), and between corneal sensitivity and total number of nerve fibers ( $r=0.55$ in PCG, $r=0.56$ in controls, all $P<0.05)$.

Finally, patients with PCG had significantly higher density of corneal basal epithelial cells $\left(6722 \pm 574\right.$ cells $\left./ \mathrm{mm}^{2}\right)$ compared with the control group $\left(6212 \pm 526\right.$ cells $\left./ \mathrm{mm}^{2}\right)(P=0.022)$, whereas the difference in the density of superficial epithelial cells was not statistically significant $\left(1892 \pm 514\right.$ cells $/ \mathrm{mm}^{2}$ in the PCG group, $2038 \pm 604$ cells $/ \mathrm{mm}^{2}$ in the control group, $P=0.11)$

\section{Discussion}

The corneal subbasal nerve plexus is a rich network of nerves located between the basal epithelium of the cornea and Bowman's layer. Corneal innervation provides protective and trophic functions, regulates epithelial integrity, proliferation, and wound healing. 8,10 Its complex stromal and epithelial branching is not visible by conventional slit-lamp biomicroscopy, but it can be visualized using in vivo confocal microscopy.

Corneal subbasal nerve plexus appears with the aid of confocal microscopy as a dense neural plexus characterized by tortuous, thin nerve fibers, with homogeneous reflectivity that is distinct from the background. ${ }^{24}$ The enhanced image brightness and contrast of LSCM allows a qualitative evaluation of certain subtle features of the cornea. ${ }^{25}$ Kobayashi et al ${ }^{26}$ first reported the presence of microstructures (called K-structures) observed at the level of Bowman's layer in normal subjects with the aid of LSCM, which were not evident with slit scanning confocal microscopy, suggesting that LSCM offers considerably higher image quality compared with conventional white-light confocal microscopy.

Several studies in normal human corneas, as well as in patients with dry eye syndrome, keratoconus, and 

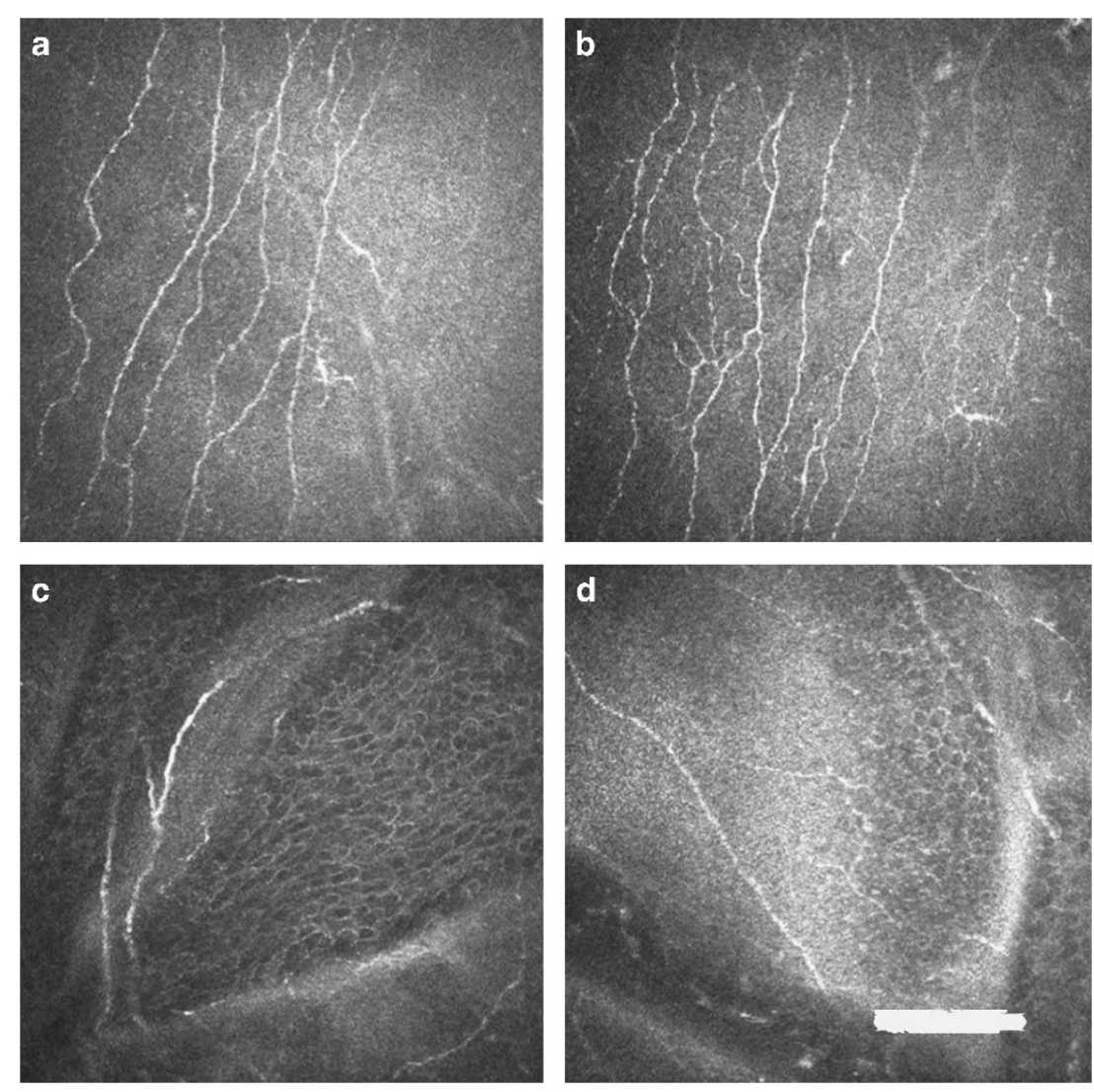

Figure 1 LSCM of the cornea. Nerve fibers of the corneal subbasal nerve plexus in control cornea (a and b). Nerve fibers of the corneal subbasal nerve plexus in congenital glaucoma (c and d). Mean subbasal nerve density was significantly decreased in congenital glaucoma $(2108 \pm 692 \mu \mathrm{m})$ compared with control cornea $(2642 \pm 484 \mu \mathrm{m})(P=0.003$, Student's $t$-test). The total number of nerve fibers in congenital glaucoma (12.3 \pm 4.2$)$ was also significantly reduced compared with control cornea $(15.4 \pm 3.1)(P=0.02$, Student's $t$-test). Scale bar represents $100 \mu \mathrm{m}$.

Table 2 Morphological analysis of corneal subbasal nerve plexus by in vivo laser-scanning confocal microscopy

\begin{tabular}{lccc}
\hline Parameters & \multicolumn{3}{c}{ Study groups } \\
\cline { 2 - 3 } & PCG $(\mathrm{n}=40)$ & Controls $(\mathrm{n}=40)$ & P-values \\
\hline Mean subbasal nerve density $(\mu \mathrm{m})$ & $2108 \pm 692$ & $2642 \pm 484$ & 0.003 \\
Total number of nerve fibers $(n)$ & $12.3 \pm 4.2$ & $15.4 \pm 3.1$ & 0.02 \\
Grade of tortuosity & $1.9 \pm 0.4$ & $1.7 \pm 0.2$ & 0.1 \\
\hline
\end{tabular}

Abbreviation: PCG, primary congenital glaucoma.

Student's $t$-test was applied. $P$-values $<0.05$ were considered statistically significant.

diabetes, have documented a positive correlation between corneal subbasal innervation and corneal sensitivity. ${ }^{13-18}$ Furthermore, a positive association between corneal nerve regeneration and corneal sensitivity has been observed after penetrating keratoplasty and laser refractive surgery. ${ }^{19-21}$

Rosenberg et $a l^{27}$ have reported that reduction of subbasal plexus nerve fibers correlates with loss of corneal sensitivity in patients with diabetic neuropathy, while tortuosity of subbasal nerves correlated significantly with the severity of diabetic neuropathy. Hamrah et $a^{28}$ have recently described significant morphological changes in corneal subbasal nerve plexus in patients with herpes simplex keratitis and showed that reduction of nerve density correlated significantly with loss of corneal sensitivity in such patients.

Mastropasqua et $a l^{7}$ in their report of two patients with PCG observed, with the aid of scanning slit corneal confocal microscope, that corneal subbasal nerve plexus 
was clearly visualized with evident thin and reflective nerve fibers and without abnormal branching. ${ }^{7}$ However, the small number of patients did not allow a quantitive analysis of the nerve plexus morphology.

In our study, we investigated the morphology of corneal subbasal nerve plexus in young patients with PCG and demonstrated that both the nerve density $(2108 \pm 692 \mu \mathrm{m})$ and the total number of nerve fibers $(12.3 \pm 4.2)$ are significantly decreased. On the other hand, nerve fiber tortuosity and corneal sensitivity are not significantly altered. No significant differences in corneal sensitivity are explained by the fact that abnormal corneal sensitivity is noted with a subbasal nerve density below the threshold of $835 \mu \mathrm{m}$ per frame. ${ }^{28}$

Furthermore, patients with PCG had significantly reduced central corneal thickness and significantly higher density of corneal basal epithelial cells compared with controls. It has been well established that corneal nerves have important trophic effects on epithelial cell growth and proliferation, keratocyte homeostasis, and corneal wound healing. ${ }^{29}$ Our results indicate that decreased corneal thickness, loss of corneal transparency, and modified corneal tissue homeostasis that are observed in PCG may be attributed not only to the globe enlargement but also to the significantly reduced corneal nerve density, at least to some extent.

Our study has certain limitations. First of all, we evaluated only the central cornea and therefore extrapolation of our results to the peripheral cornea would not be reasonable. Moreover, the Cochet-Bonnet esthesiometer is limited to the evalulation of mechanical nociceptors. ${ }^{30}$ Nevertheless, it still remains a widely tested, valid modality for the evaluation of corneal sensitivity, despite its drawbacks. ${ }^{31,32}$

Another limitation derives from the fact that the majority of the patients with PCG examined in this study received topical antiglaucomatous therapy (carboanhydrase inhibitors and/or brimonidine eye drops). It has been reported that chronic topical treatment with timolol and latanoprost may induce toxic effects on ocular surface, leading to reduction of the corneal subbasal nerve density, thereby affecting corneal sensitivity. ${ }^{33}$ Moreover, Baratz et al ${ }^{34}$ have documented that chronic administration of glaucoma medication in patients with ocular hypertension causes a decrease in the corneal subbasal nerve density without affecting keratocyte density or corneal endothelial characteristics. However, it has been suggested that ocular surface changes induced by timolol and latanoprost are mainly attributed to the preservatives, ${ }^{33}$ whereas Baratz et al ${ }^{34}$ evaluated a small sample size $(n=27)$ with 10 different glaucoma medications, which did not allow any subgroup analysis, thereby preventing the authors from implicating specific medications or preservatives, as also indicated by the authors themselves. ${ }^{34}$ Nevertheless, although there is no definitive proof that carboanhydrase inhibitors or brimonidine eye drops affect the corneal subbasal nerve density, one could not exclude such an effect.

In conclusion, to our knowledge, this is the first study to report on the morphological alterations of corneal subbasal nerve plexus in PCG and their impact on corneal sensitivity, using LSCM. Further studies with larger cohorts of patients are necessary to investigate the corneal subbasal nerve plexus and reveal the complex nature of human corneal sensitivity.

\section{Summary}

\section{What was known before}

- There are only two case reports of patients with congenital glaucoma who displayed an abnormal, 'coil-shaped' configuration of the corneal nerve fiber network.

What this study adds

- This study investigates the alterations in the corneal subbasal nerve plexus in congenital glaucoma in a validated and quantitative manner.

- Primary congenital glaucoma exerts significant changes in both the mean subbasal nerve density and the total number of nerve fibers, but without affecting corneal sensitivity.

\section{Conflict of interest}

The authors declare no conflict of interest.

\section{References}

1 Beck AD. Diagnosis and management of pediatric glaucoma. Ophthalmol Clin N Am 2001; 14: 501-512.

2 Cibis GW, Tripathi RC. The differential diagnosis of Descemet's tears (Haab's striae) and posterior polymorphous dystrophy bands. A clinicopathological study. Ophthalmology 1982; 89: 614-620.

3 Ho CL, Walton DS. Primary congenital glaucoma: 2004 update. J Pediatr Ophthalmol Strabismus 2004; 41: 271-288.

4 Gatzioufas Z, Labiris G, Stachs O, Hovakimyan M, Schnaidt A, Viestenz A et al. Biomechanical profile of the cornea in primary congenital glaucoma. Acta Ophthalmol 2013; 91(1): 29-34.

5 Khan AO. Genetics of primary glaucoma. Curr Opin Ophthalmol 2001; 22: 347-355.

6 deLuise VP, Anderson DR. Primary infantile glaucoma (congenital glaucoma). Surv Ophthalmol 1983; 28: 1-19.

7 Mastropasqua L, Carpineto P, Ciancaglini M, Nubile M, Doronzo E. In vivo confocal microscopy in primary congenital glaucoma with megalocornea. J Glaucoma 2002; 11: 83-89.

8 Guthoff RF, Wienss H, Hahnel C, Wree A. Epithelial innervation of human cornea: a three-dimensional study using confocal laser scanning fluorescence microscopy. Cornea 2005; 24: 608-613. 
9 Patel DV, McGhee CN. In vivo confocal microscopy of human corneal nerves in health, in ocular and systemic disease, and following corneal surgery: a review. Br J Ophthalmol 2009; 93: 853-860.

10 Oliveira-Soto L, Efron N. Morphology of corneal nerves using confocal microscopy. Cornea 2001; 20: 374-384.

11 Grupcheva CN, Wong T, Riley AF, McGhee CN. Assessing the sub-basal nerve plexus of the living healthy human cornea by in vivo confocal microscopy. Clin Exp Ophthalmol 2002; 30: 187-190.

12 Patel DV, McGhee CN. Mapping of the normal human corneal sub-basal nerve plexus by in vivo laser scanning confocal microscopy. Invest Ophthalmol Vis Sci 2005; 46: 4485-4488.

13 Patel DV, McGhee CN. Mapping the corneal sub-basal nerve plexus in keratoconus by in vivo laser scanning confocal microscopy. Invest Ophthalmol Vis Sci 2006; 47: 1348-1351.

14 Simo Mannion L, Tromans C, O'Donnell C. An evaluation of corneal nerve morphology and function in moderate keratoconus. Contact Lens Anterior Eye 2005; 28: 185-192.

15 Tuominen IS, Konttinen YT, Vesaluoma MH, Moilanen JA, Helintö M, Tervo TM. Corneal innervation and morphology in primary Sjögren's syndrome. Invest Ophthalmol Vis Sci 2003; 44: 2545-2549.

16 Benitez del Castillo JM, Wasfy MA, Fernandez C, Garcia-Sanchez J. An in vivo confocal masked study on corneal epithelium and subbasal nerves in patients with dry eye. Invest Ophthalmol Vis Sci 2004; 45: 3030-3035.

17 Hosal BM, Ornek N, Zilelioglu G, Elhan AH. Morphology of corneal nerves and corneal sensation in dry eye: a preliminary study. Eye (Lond) 2005; 19: 1276-1279.

18 Patel DV, Ku JY, Johnson R, McGhee CN. Laser scanning in vivo confocal microscopy and quantitative aesthesiometry reveal decreased corneal innervation and sensation in keratoconus. Eye (Lond) 2009; 23: 586-592.

19 Niederer RL, Perumal D, Sherwin T, McGhee CN. Corneal innervation and cellular changes after corneal transplantation: an in vivo confocal microscopy study. Invest Ophthalmol Vis Sci 2007; 48: 621-626.

20 Calvillo MP, McLaren JW, Hodge DO, Bourne WM. Corneal reinnervation after LASIK: prospective 3-year longitudinal study. Invest Ophthalmol Vis Sci 2004; 45: 3991-3996.

21 Erie JC, McLaren JW, Hodge DO, Bourne WM. Recovery of corneal subbasal nerve density after PRK and LASIK. Am J Ophthalmol 2005; 140: 1059-1064.

22 Stave J, Zinser G, Grümmer G, Guthoff RF. Der modifizierte Heidelberg-Retina-Tomograph HRT. Erste Ergebnisse einer In-vivo-Darstellung von kornealen Strukturen. Ophthalmologe 2002; 99: 276-280.

23 Benitez del Castillo JM, Acosta MC, Wassfi MA, Diaz-Valle D, Gegundez JA, Fernandez C et al. Relation between corneal innervation with confocal microscopy and corneal sensitivity with noncontact esthesiometry in patients with dry eye. Invest Ophthalmol Vis Sci 2007; 48: 173-181.

24 Villani E, Galimberti D, Viola F, Mapelli C, Ratiglia R. The cornea in Sjogren's syndrome: an in vivo confocal study. Invest Ophthalmol Vis Sci 2007; 48: 2017-2022.

25 Patel DV, McGhee CN. Contemporary in vivo confocal microscopy of the living human cornea using white light and laser scanning techniques: a major review. Clin Exp Ophthalmol 2007; 35: 71-88.

26 Kobayashi A, Yokogawa H, Sugiyama K. In vivo laser confocal microscopy of Bowman's layer of the cornea. Ophthalmology 2006; 113: 2203-2208.

27 Rosenberg ME, Tervo TM, Immonen IJ, Muller LJ, Gronhagen-Riska C, Vesaluoma MH. Corneal structure and sensitivity in type 1 diabetes mellitus. Invest Ophthalmol Vis Sci 2000; 41: 2915-2921.

28 Hamrah P, Cruzat A, Dastjerdi MH, Zheng L, Shahatit BM, Bayhan HA et al. Corneal sensation and subbasal nerve alterations in patients with herpes simplex keratitis: an in vivo confocal microscopy study. Ophthalmology 2010; 117: 1930-1936.

29 Müller LJ, Marfurt CF, Kruse F, Tervo TM. Corneal nerves: structure, contents and functions. Exp Eye Res 2003; 76(5): 521-542.

30 Patel DV, Tavakoli M, Craig JP, Efron N, McGhee CN. Corneal sensitivity and slit scanning in vivo confocal microscopy of the subbasal nerve plexus of the normal central and peripheral human cornea. Cornea 2009; 28: 735-740.

31 Zhivov A, Winter K, Hovakimyan M, Peschel S, Harder V, Schober HC et al. Imaging and quantification of subbasal nerve plexus in healthy volunteers and diabetic patients with or without retinopathy. PLoS One 2013; 8(1): e52157.

32 Labbé A, Alalwani H, Van Went C, Brasnu E, Georgescu D, Baudouin $\mathrm{C}$. The relationship between subbasal nerve morphology and corneal sensation in ocular surface disease. Invest Ophthalmol Vis Sci 2012; 53(8): 4926-4931.

33 Martone G, Frezzotti P, Tosi GM, Traversi C, Mittica V, Malandrini A et al. An in vivo confocal microscopy analysis of effects of topical antiglaucoma therapy with preservative on corneal innervation and morphology. Am J Ophthalmol 2009; 147(4): 725-735.

34 Baratz KH, Nau CB, Winter EJ, McLaren JW, Hodge DO, Herman DC et al. Effects of glaucoma medications on corneal endothelium, keratocytes, and subbasal nerves among participants in the ocular hypertension treatment study. Cornea 2006; 25(9): 1046-1052. 